\title{
64. Preliminary Report of Studies on Genetic Effects of Consanguineous Marriages. III*)
}

\author{
Ascertainment and Estimation of Mortality - a \\ Consanguinity Study in Shizuoka
}

By Katumi TANAKA**)

(Comm. by Taku KomaI, M.J.A., April 13, 1964)

Introduction. It is well known that biases may be introduced into genetic data through the method by which families including affected individuals come to the investigators' attention. The problems concerning ascertainment have been discussed by various authors (Bailey, 1951; Fisher, 1934, 1936; Schull, 1954; Morton, 1959; etc.) particularly with regard to the estimation of recombination values, segregation ratios, and gene frequencies.

Our consanguinity study team ${ }^{1)}$ under the chairmanship of Dr. Komai came to the tentative conclusion that the mortality rate among children of related parents is higher than that among those of unrelated parents..$^{2,3)}$ However, closer study of the data has revealed that the method of ascertainment may have introduced a bias into the estimation of the two mortality rates. The nature of this bias is set forth in the present report.

Method of obtaining information on mortality. In the summers of 1958, 1959, and 1960, children of 12 primary schools in Shizuoka City were subjected to anthropometric, psychometric, and medical examinations. In addition, information on fetal and postnatal deaths among the siblings of these children was collected for the purpose of the comparative study of the rate of such deaths between consanguineous and non-consanguineous families.

In the selection of these families two different methods were adopted. In the study in 1958 all children in 2 primary schools were chosen as propositi, irrespective of parental relationship. Accordingly, almost all of the families with at least one child attending any of these schools were included in the sample.

In 1959 and 1960, on the other hand, while all children born to consanguineous couples were selected as propositi, only a random sam-

*) Cf. Ibid. I. Proc. Japan Acad., 39(6): 380-384, and Ibid. II. 39(7): 497-502. Part of research was supported by grants from the Ministry of Education (Sci. Res. Project No. 9112 in 1958 and 1959, No. 9109 in 1960, No. 0427 in 1961 and No. 0414 in 1962) and the Rockefeller Foundation (RF 59149). c/o Publication Center, Consanguinity Study.

**) Department of Human Genetics, Tokyo Medical and Dental University, Bunkyoku, Tokyo. 
ple of the children born to non-consanguineous couples were so selected. The number of propositi in the control group was adjusted to be approximately equal to that of the consanguineous group. Thus, all the consanguineous families, but only a part of the non-consanguineous families, were included in the sample. The number of families examined was somewhat larger in the control group than in the consanguineous group, because a greater number of families having more than one propositus were included in the latter group than in the former group.

To obtain an exact mortality rate, the ages of the sibs of the propositi must be taken into account. For instance, mortality in a certain period, say, 1 to 5 years of age, should mean the frequency of death in the period 1-5 years among children who were born 6 years or more previously, and had survived at least one year. The method of ascertainment may introduce some bias in the estimation of mortality in the age groups containing the propositi, but not in that of other age groups, if it is assumed that the number of school age children is not correlated with mortality among their sibs.

Relationships between the real and the apparent death rate. As mentioned above, we propose to estimate two mortality rates from a study on the mortality of siblings in two groups of surviving children. The situation is different from that of ordinary incomplete ascertainment in which sibships are selected via affected children. Thus, a somewhat different method is required.

Let $s$ be the number of children born in the period 6-11 years previously, $N_{s}$ the number of families containing such children, $q$ the probability of death in the interval 0-5 years, $p$ the probability that a child will survive to 6 years $(=1-q), v$ the number of surviving children in a family, and $r$ the number of children who died at ages 0-5 years $(v+r=s)$, then the probability of a given family of size $s$ containing $v$ surviving children is $\left(\begin{array}{c}s \\ r\end{array}\right) p^{s-r} q^{r}$, excluding families of $s-r=0$ or $s=r$.

Let $\pi=p_{1} / p$ be the probability that a surviving child becomes a proband, and $1-\pi=p_{2} / p$ the probability that he (or she) does not $\left(p_{1}+p_{2}=p\right.$ and $\left.p_{1}+p_{2}+q=1\right)$. The probability that a family having $v$ surviving children is not ascertained is $(1-\pi)^{v}$ or $(1-\pi)^{s-r}$ and the probability that the family is ascertained is $1-(1-\pi)^{s-r}$ or $\left(p^{s-r}-\right.$ $\left.p_{2}{ }^{s-r}\right) / p^{s-r}$. Hence, the expected number of families in which $r$ of $s$ children are dead is

$$
N_{s}\left[\left(\begin{array}{l}
s \\
r
\end{array}\right) p^{s-r} q^{r}\right]\left(p^{s-r}-p_{2}{ }^{s-r}\right) / p^{s-r} \text { or } N_{s}\left[\left(\begin{array}{l}
s \\
r
\end{array}\right) q^{r}\left(p^{s-r}-p_{2}{ }^{s-r}\right)\right] .
$$

The total number of children contained in these ascertained families is 
No. 4]

$$
\begin{aligned}
& s N_{s} \sum_{r=0}^{s-1}\left(\begin{array}{c}
s \\
r
\end{array}\right) q^{r}\left(p^{s-r}-p_{2}{ }^{s-r}\right) \\
= & s N_{s}\left\{\sum\left(\begin{array}{c}
s \\
r
\end{array}\right) p^{s-r} q^{r}-\sum\left(\begin{array}{c}
s \\
r
\end{array}\right) p_{2}{ }^{s-r} q^{r}\right\} \\
= & s N_{s}\left[(p+q)^{s}-q^{s}-\left\{\left(p_{2}+q\right)^{s}-q^{s}\right\}\right] \\
= & s N_{s}\left\{1-\left(p_{2}+q\right)^{s}\right\} \\
= & s N_{s}\left\{1-\left(1-p_{1}\right)^{s}\right\}
\end{aligned}
$$

and the total number of dead children contained in the ascertained families is

$$
\begin{aligned}
& N_{s} \sum_{r=0}^{s-1}\left(\begin{array}{l}
s \\
r
\end{array}\right) q^{r} r\left(p^{s-r}-p_{2}^{s-r}\right) \\
& \left.=N_{s}\left\{\begin{array}{l}
s \\
r
\end{array}\right) p^{s-r} q^{r} r-\sum\left(\begin{array}{c}
s \\
r
\end{array}\right) p_{2}^{s-r} q^{r} r\right\} \\
& =s q N_{s}\left\{\sum\left(\begin{array}{l}
s-1 \\
r-1
\end{array}\right) p^{(s-1)-(r-1)} q^{r-1}-\sum\left(\begin{array}{c}
s-1 \\
r-1
\end{array}\right) p_{2}^{(s-1)-(r-1)} q^{r-1}\right\} \\
& =s q N_{s}\left[(p+q)^{s-1}-q^{s-1}-\left\{\left(p_{2}+q\right)^{s-1}-q^{s-1}\right\}\right] \\
& =s q N_{s}\left\{1-\left(p_{2}+q\right)^{s-1}\right\} \\
& =s q N_{s}\left\{1-\left(1-p_{1}\right)^{s-1}\right\} .
\end{aligned}
$$

Hence, an apparent mortality, $q^{\prime}$, calculated by using the data of ascertained families only is $q^{\prime}=q\left\{1-\left(1-p_{1}\right)^{s-1}\right\} /\left\{1-\left(1-p_{1}\right)^{s}\right\}$. Since $0 \leq\left(1-p_{1}\right) \leq 1,\left(1-p_{1}\right)^{s-1}$ is always larger than $\left(1-p_{1}\right)^{s}$ unless $p_{1}$ is 0 or 1 , and the apparent mortality, $q^{\prime}$, will be smaller than the true value, $q$.

The ratio $q^{\prime} / q$, is a function of $p_{1}$ and $s$. With larger $s$ the ratio approximates to unity more closely. If $s=1, q^{\prime}$ should become 0 . Likewise, with a smaller $p_{1}, q^{\prime} / q$ becomes smaller. Numerical examples of these relations are given in Table $\mathrm{I}$.

Table I. Ratio of the apparent mortality to the true one for various values of sibship size and probabilities of ascertainment

\begin{tabular}{l|c|c|c|c|c|c}
\hline$p_{1}$ & $p_{2}+q$ & $s=1$ & $s=2$ & $s=3$ & $s=4$ & $s=5$ \\
\hline 0.9 & 0.1 & 0 & 0.9091 & 0.9910 & 0.9991 & 0.9999 \\
0.7 & 0.3 & 0 & 0.7692 & 0.9353 & 0.9809 & 0.9943 \\
0.5 & 0.5 & 0 & 0.6667 & 0.8571 & 0.9333 & 0.9677 \\
0.3 & 0.7 & 0 & 0.5882 & 0.7763 & 0.8646 & 0.9134 \\
0.1 & 0.9 & 0 & 0.5263 & 0.7011 & 0.7880 & 0.8398 \\
\hline
\end{tabular}

In the foregoing discussion families of equal size are considered. If data for different family sizes are pooled, the apparent mortality might be calculated as

$$
q^{\prime}=q \sum_{s=1} s N_{s}\left\{1-\left(1-p_{1}\right)^{s-1}\right\} / \sum_{s=1} s N_{s}\left\{1-\left(1-p_{1}\right)^{s}\right\}
$$

Formula (3) indicates that the proportion of families of different sizes in the sample affects the estimation of the apparent mortality rate; that is, the smaller the proportion of large families, the lower 
becomes the estimate. Since, the apparent mortality is 0 in ascertained one-child families irrespective of the true mortality, inclusion of such families in the sample should considerably decrease the apparent mortality rate and obscure existing differences in real mortality rates among populations or groups.

Table II. Probability that a family is ascertained, $1-\left(1-p_{1}\right)^{s}$, for various values of sibship size and probability of ascertainment

\begin{tabular}{l|c|c|c|c|c|c}
\hline$p_{1}$ & $p_{2}+q$ & $s=1$ & $s=2$ & $s=3$ & $s=4$ & $s=5$ \\
\hline 0.9 & 0.1 & 0.9 & 0.99 & 0.999 & 0.9999 & 0.99999 \\
0.7 & 0.3 & 0.7 & 0.91 & 0.973 & 0.9919 & 0.99757 \\
0.5 & 0.5 & 0.5 & 0.75 & 0.875 & 0.9375 & 0.96875 \\
0.3 & 0.7 & 0.3 & 0.51 & 0.657 & 0.7599 & 0.83193 \\
0.1 & 0.9 & 0.1 & 0.19 & 0.271 & 0.3439 & 0.40951 \\
\hline
\end{tabular}

On the other hand, the probability that a family is ascertained, $1-\left(1-p_{1}\right)^{s}$, increases with increasing $s$ and $p_{1}$ (Table II). As stated above, the decrease of $p_{1}$ would result in an increase in the difference between apparent and real mortality rates if families of different sizes are considered separately. This difference, however, may be compensated for in pooled data by an increase in the proportion of large families.

Effect of the method of ascertainment on the estimate of mortality in Shizuoka samples. As stated above, in the 2 primary schools studied in 1958, almost all children, inbred or not, were selected as propositi, and $p_{2}$ is 0 in both consanguineous and unrelated groups. As far as families of equal sizes are concerned, no difference in sampling procedure would exist between the two groups. Any difference observed in the apparent mortality could readily be accepted as an evidence of a difference in real mortality, unless the two groups differed substantially in the distribution of family sizes.

As for the other 10 schools, $p_{2}=0$ in the consanguineous group, while $p_{2}>0$ in the control group. Accordingly, the apparent mortality in the former group should be higher than that in the control group, even if the true rate is the same. Since numbers of propositi in the control groups are approximately equal to those in the consanguineous groups, the probability that non-inbred children become propositi is proportional to the consanguinity rate. Consequently, the value $p_{1}$ in the control group is larger and the decrease in apparent mortality would be smaller in localities of high consanguinity than in localities of lower consanguinity. All these considerations apply to fetal deaths.

Estimation of the true mortality rate. Since the probability that a child becomes a propositus is $p_{1}$, the probability that he does not 
is $1-p_{1}$. Hence, the probability that none of $s$ children is selected as a propositus is $\left(1-p_{1}\right)^{s}$, and the probability that a family of size $s$ is ascertained is $1-\left(1-p_{1}\right)^{s}$. Let $N_{s}^{\prime}$ be the number of ascertained families, then, the total number of families of size $s$ would be $N_{s}=N_{s}^{\prime} /\left\{1-\left(1-p_{1}\right)^{s}\right\}$. Let $t$ be the number of propositi in a family and $A_{t s}$ the number of families in which $t$ of $s$ children are propositi. The expected number of $A_{t s}$ family is

$$
E\left(A_{t s}\right)=N_{s}^{\prime}\left(\begin{array}{l}
s \\
t
\end{array}\right) p_{1}^{t}\left(1-p_{1}\right)^{s-t} /\left\{1-\left(1-p_{1}\right)^{s}\right\} .
$$

The maximum likelihood function is

$$
L=\prod_{s} \prod_{t}\left[\frac{1}{1-\left(1-p_{1}\right)^{s}}\left(\begin{array}{l}
s \\
t
\end{array}\right) p_{1}^{t}\left(1-p_{1}\right)^{s-t}\right]^{A_{t s}}
$$

$\log L=\sum_{s} \sum_{t}\left[t \log p_{1}+(s-t) \log \left(1-p_{1}\right)-\log \left\{1-\left(1-p_{1}\right)^{s}\right\}\right] A_{t s}+$ const.

$$
\begin{aligned}
\frac{d(\log L)}{d p_{1}} & =\sum_{s} \sum_{t}\left[\frac{t}{p_{1}}-\frac{s-t}{1-p_{1}}-\frac{s\left(1-p_{1}\right)^{s-1}}{1-\left(1-p_{1}\right)^{s}}\right] A_{t s} \\
& =\sum_{s} \sum_{t} \frac{t A_{t s}}{p_{1}}-\sum_{s} \sum_{t}\left[\frac{s-t\left\{1-\left(1-p_{1}\right)^{s}\right\}}{\left(1-p_{1}\right)\left\{1-\left(1-p_{1}\right)^{s}\right\}}\right] A_{t s}=0 \\
\frac{1}{p_{1}} \sum_{s} \sum_{t} t A_{t s} & =\frac{1}{1-p_{1}} \sum \sum \frac{s A_{t s}}{1-\left(1-p_{1}\right)^{s}}-\frac{1}{1-p_{1}} \sum \sum t A_{t s} .
\end{aligned}
$$

$\sum \sum t A_{t s}$ is the total number, say $T$, of propositi in ascertained families. $\sum_{t} A_{t s}$ is the total number of ascertained families of size $s$, i.e. $N_{s}^{\prime}$. The last equation reduces to

$$
\frac{T}{p_{1}}=\sum_{s} \frac{s N_{s}^{\prime}}{1-\left(1-p_{1}\right)^{s}} .
$$

As $T$ and $s N_{s}^{\prime}$ are known, the maximum likelihood estimate of $p_{1}$ may be obtained by trial and error. The families containing only one child (i.e. the propositus) should be excluded since they would give no information on mortality. There are few families which contain 4 or more children born 6-11 years ago. Thus, most families usable for the calculation contain 2 or 3 children. This situation will facilitate the calculation.

Since $N_{s}=N_{s}^{\prime} /\left\{1-\left(1-p_{1}\right)^{s}\right\}$, Formula (3) may be transformed into

$$
q=q^{\prime} \sum_{s=2} s N_{s}^{\prime} / \sum_{s=2} s N_{s}^{\prime}\left\{\frac{1-\left(1-p_{1}\right)^{s-1}}{1-\left(1-p_{1}\right)^{s}}\right\} \text {. }
$$

Putting the $p_{1}$ value into Formula (4) one obtains an estimate of the true mortality.

The estimate thus derived is the total rate of individuals who died before the age of 6 years. Since the probability that a dead child is ascertained would not be affected by his (or her) age at death, the true mortality for a given age period, fetal, neonatal, infantile stages, etc., may be estimated by multiplying the apparent mortality for the period by $q / q^{\prime}$. 
The results of actual calculation of mortality rates by the method set forth above will be presented in a subsequent report in this series.

The author is greatly indebted to Drs. T. Komai, W. J. Schull, M. Kimura, E. Matsunaga, A. Kudo, and T. Yanase who have critically commented on the manuscript.

\section{References}

Komai, T. (1963): Preliminary reports of studies on genetic effect of consanguineous marriages. I. Proc. Japan Acad., 39, 380-384.

Kishimoto, K. (1962): Preliminary report of activities of the consanguinity study group of the Science Council of Japan. Eugenics Quart., 9, 5-13.

Tanaka, K. (1963): Consanguinity study on Japanese population. The Genetics of Migrant and Isolate Populations (E. Goldschmidt, ed.), 169-176. 